\title{
Testing the Field Correlator Method with astrophysical constraints
}

\section{G. F. Burgio*}

INFN Sezione di Catania, Via S. Sofia 64, I-95123 Catania, Italy

E-mail: fiorella.burgiodct.infn.it

\section{Baldo}

INFN Sezione di Catania, Via S. Sofia 64, I-95123 Catania, Italy

E-mail: marcello.baldoect.infn.it

\section{P. Castorina}

Dipartimento di Fisica e Astronomia, Universita' di Catania, and INFN Sezione di Catania, Via

S. Sofia 64, I-95123 Catania, Italy

E-mail: paolo.castorinaect.infn.it

\section{S. Plumari}

Dipartimento di Fisica e Astronomia, Universita' di Catania, and INFN Sezione di Catania, Via

S. Sofia 64, I-95123 Catania, Italy

E-mail: salvatore.plumariect.infn.it

\section{Zappala'}

INFN Sezione di Catania, Via S. Sofia 64, I-95123 Catania, Italy

E-mail: dario.zappala@ct.infn.it

We study the structure of hybrid stars with the Field Correlator Method, extended to the zero temperature limit, for the quark phase. For the hadronic phase, we use the microscopic BruecknerHartree- Fock many-body theory. The comparison with the neutron star mass phenomenology puts serious constraints on the currently adopted values of the gluon condensate $G_{2} \simeq 0.006-$ $0.007 \mathrm{GeV}^{4}$, and the large distance static $Q \bar{Q}$ potential.

8th Conference Quark Confinement and the Hadron Spectrum September 1-6 2008

Mainz, Germany

\footnotetext{
*Speaker.
} 


\section{Introduction}

The study of the properties of neutron stars (NS) concerns the large density (and low temperature) region of the phase diagram and, in particular, it requires the QCD non-pertubative Equation of State (EoS) at small $T$ and large $\mu_{q}$, where no QCD lattice simulations are available yet. Due to the lack of lattice data, analytic approaches based on more elementary models, such as the NambuJona-Lasinio (NJL) model [1], the MIT Bag model, and the Color Dielectric model [2] are mostly used. A serious drawback of those models is that they cannot make predictions for both limits, i.e. high temperature and zero chemical potential or high chemical potential and low temperature, and therefore cannot be fully tested. One of the few exceptions is the Field Correlator Method (FCM) [3], which in principle is able to cover the full temperature-chemical potential plane. The method contains $a b$ initio the property of confinement, which should play a role in the stability of the pure quark phase in neutron stars, as we discussed in ref.[4]. Based on that, we have tested [5] the FCM by comparing the results for the neutron star masses with the existing phenomenology, which turns out to be a strong constraint on the parameters used in the model. In particular, we have found definite numerical indications on some relevant physical quantities, such as the gluon condensate and the $Q \bar{Q}$ potential, to be compared to the ones extracted from the determination of the critical temperature of the deconfinement phase transition. This shows the relevance of the comparison of the model predictions in the high chemical potential region with the astrophysical phenomenology.

\section{Hadronic and quark EOS}

The main point of our study is the comparison of the nuclear matter EoS with the one for quark matter. We start with the description of the hadronic phase. The EoS is based on the BruecknerBethe-Goldstone (BBG) many-body theory, which is a linked cluster expansion of the energy per nucleon of nuclear matter [6]. It has been shown that the non-relativistic BBG expansion is well convergent, and the Brueckner-Hartree-Fock (BHF) level of approximation is accurate in the density range relevant for neutron stars. In the BHF approximation the energy per nucleon is

$$
\frac{E}{A}=\frac{3}{5} \frac{k_{F}^{2}}{2 m}+\frac{1}{2 n} \operatorname{Re} \sum_{k, k^{\prime} \leq k_{F}}\left\langle k k^{\prime}\left|G\left[n ; e(k)+e\left(k^{\prime}\right)\right]\right| k k^{\prime}\right\rangle_{a} .
$$

where $G$ is the Brueckner reaction matrix, which contains the bare nucleon-nucleon (NN) interaction, and the nucleon number density $n . e(k)$ is the single-particle energy, and the subscript " $a$ " indicates antisymmetrization of the matrix element. In the calculations reported here we have used the Argonne $v_{18}$ potential as the two-nucleon interaction, supplemented by three-body forces built according to the Urbana model [7]. The corresponding nuclear matter EOS reproduces correctly the nuclear matter saturation point $\rho_{0}=0.17 \mathrm{fm}^{-3}, E / A=-16 \mathrm{MeV}$ [8]. In neutron stars one has to consider matter in beta equilibrium, where electrons and eventually muons coexist with baryons, while neutrinos are considered to escape from the star. The EOS for the beta equilibrated matter can be obtained once the hadron matter is known, together with the chemical potentials of different species as a function of total baryon density. Since the procedure is standard, we do not give further details of the calculations. 
Let us now illustrate the EoS for quark matter. A systematic method to treat non perturbative effects in QCD is by gauge invariant field correlators [3]. The approach based on the FCM provides a natural treatment of the dynamics of confinement (and of the deconfinement transition) in terms of the color electric and color magnetic Gaussian correlators. At $\mu_{q}=0$, the analytical results are in reasonable agreement with lattice data [9, 10, 11]. The extension in ref. [10] of the FCM to finite values of the chemical potential, allows to obtain a simple expression of the Equation of State of the quark-gluon matter, which reads

$$
P_{q g}=P_{g}+\sum_{j=u, d, s} P_{q}^{j}+\Delta \varepsilon_{v a c}
$$

where $P_{g}$ and $P_{q}^{j}$ are respectively the gluon and quark pressure, and

$$
\Delta \varepsilon_{v a c} \approx-\frac{\left(11-\frac{2}{3} N_{f}\right)}{32} \frac{G_{2}}{2}
$$

corresponds to the difference of the vacuum energy density in the two phases, being $N_{f}$ the flavour number, and $G_{2}$ the gluon condensate, whose numerical value is known with large uncertainty, $G_{2}=0.012 \pm 0.006 \mathrm{GeV}^{4}$ [12]. Within the FCM, the quark pressure, for a single flavour, is given by $[11,13,14]$

$$
\begin{aligned}
P_{q} / T^{4} & =\frac{1}{\pi^{2}}\left[\phi_{v}\left(\frac{\mu_{q}-V_{1} / 2}{T}\right)+\phi_{v}\left(-\frac{\mu_{q}+V_{1} / 2}{T}\right)\right] \\
\phi_{v}(a) & =\int_{0}^{\infty} d u \frac{u^{4}}{\sqrt{u^{2}+v^{2}}} \frac{1}{\left(\exp \left[\sqrt{u^{2}+v^{2}}-a\right]+1\right)}
\end{aligned}
$$

where $v=m_{q} / T$, and $V_{1}$ is the large distance static $Q \bar{Q}$ potential. The gluon contribution to the pressure is

$$
P_{g} / T^{4}=\frac{8}{3 \pi^{2}} \int_{0}^{\infty} d \chi \chi^{3} \frac{1}{\exp \left(\chi+\frac{9 V_{1}}{8 T}\right)-1}
$$

The potential $V_{1}$ is assumed to be independent on the chemical potential, and this is partially supported by lattice simulations at small chemical potential [11].

The comparison between the pressures in the hadron and quark phases is shown in Fig. 1 (left panels). We adopt the simple Maxwell construction, which implies that the phase coexistence is determined by a crossing point in the pressure vs. chemical potential plot. In the upper panel we show the results obtained using $V_{1}=0$, whereas in the lower panel calculations with $V_{1}=0.01$ $\mathrm{GeV}$ are displayed. The solid line represents the calculations performed with the BBG method with nucleons, and the other lines represent results obtained with different choices of the gluon condensate $G_{2}$. The chosen values of $G_{2}$ give values of the critical temperature in a range between 160 and $190 \mathrm{MeV}$ [11]. We observe i) the crossing point is significantly affected by the value of the gluon condensate, and only slightly by the chosen value of the potential $V_{1}$, ii) with increasing $G_{2}$, the onset of the phase transition is shifted to larger chemical potentials. In Fig. 1 (right panels) we display the pressure vs. nucleon density relationship, for the several cases shown in the corresponding left panels. Below the plateau, $\beta$-stable and charge neutral stellar matter is in the purely hadronic phase, whereas for density above the ones characterizing the plateau, the system is in the pure quark phase. 

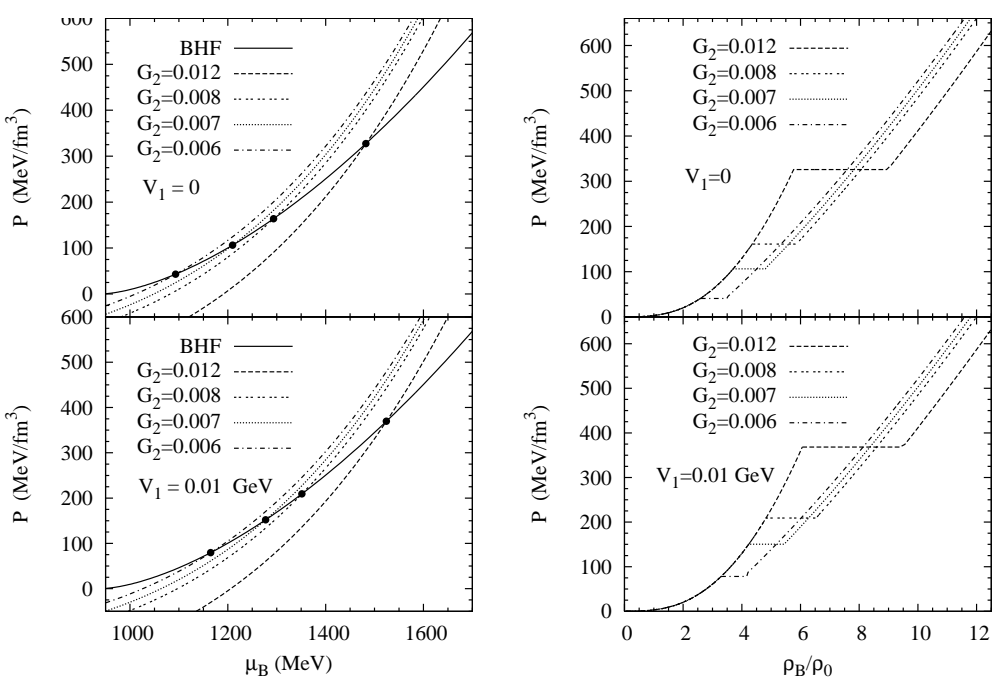

Figure 1: The pressure vs. baryon chemical potential (density normalized with respect to the saturation value) is displayed in left (right) plots for $V_{1}=0$ (upper panels), and $V_{1}=0.01$ (lower panels).

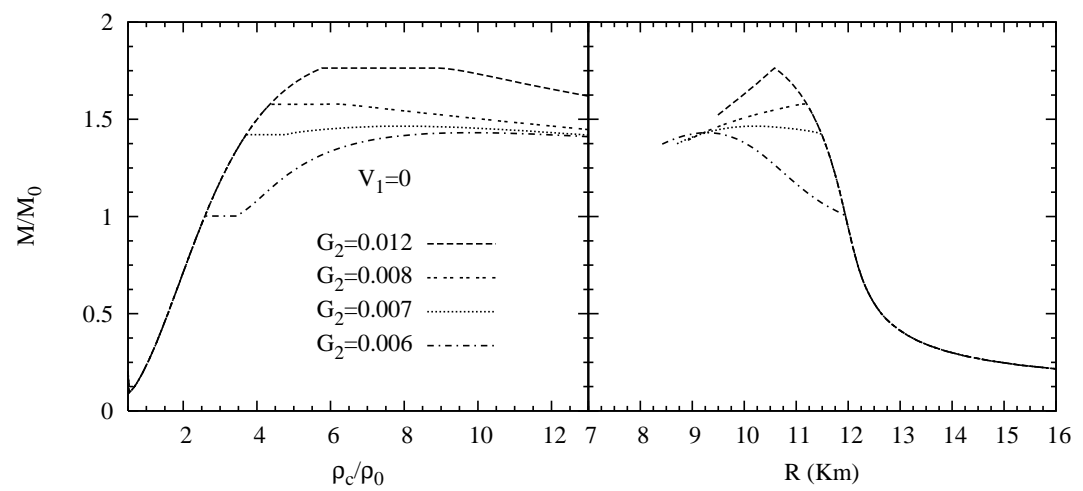

Figure 2: The gravitational mass (in units of the solar mass) is displayed as a function of the central baryon density, normalized with respect to the nuclear matter saturation density $\rho_{0}$ (left panel), and the corresponding radius (right panel).

The EoS is the fundamental input for solving the hydrostatic equilibrium equations of Tolman, Oppenheimer, and Volkov [15]. In Fig.2 (left panel) we display the gravitational mass (in units of solar mass $M_{\odot}=2 \times 10^{33} \mathrm{~g}$ ) as a function of the central baryon density (normalized with respect to the saturation value), and the corresponding radius (right panel). We observe that the value of the maximum mass spans over a range between 1.4 and 1.8 solar masses, depending on the value of the gluon condensate $G_{2}$. The stability of the pure quark phase appears only for small values of $G_{2}$, but the corresponding values of the gravitational maximum mass are hardly in agreement with observational data. In fact, we recall that any "good" equation of state must give for the maximum mass at least 1.44 solar mass, the best measured value so far [16]. By increasing the value of $G_{2}$, the maximum mass increases up to about 1.8 solar mass, but the stability of the pure quark phase is lost, and the maximum mass contains in its interior at most a mixed quark-hadron phase. We observe a similar trend even if $V_{1}>0$ [5]. However, the observational data indicate that NS with 
a mass of at least 1.6 solar masses do exist [17], and this puts a serious constraint on the value of the gluon condensate, which is not easy to reconcile with the value $0.006 \mathrm{GeV}^{4}$, extracted from the comparison with the lattice data on the critical temperature. As far as the value of the large distance static $Q \bar{Q}$ potential $V_{1}$ is concerned, in the comparison with lattice calculations [10] one finds a value $V_{1} \sim 0.5 \mathrm{GeV}$ at the critical temperature and for $\mu=0$. We have therefore changed the strength of $V_{1}$ from small values up to $0.5 \mathrm{GeV}$, and found that already for $V_{1}=100 \mathrm{MeV}$ the phase transition cannot occur in NS, which is then composed of baryon matter only, with a maximum mass around 2 solar masses. For higher values of $V_{1}$ the transition can possibly occur only at exceedingly high values of the density, and therefore the quark phase is irrelevant for NS physics.

These results indicate a direct link between the NS quark content and the properties of deconfinement in the hadron-quark phase transition. More quantitatively, if one considers that the well established values of NS masses never exceed $\approx 1.6$ solar masses, then these observational data constrain $V_{1}$ to small values and in a narrow range, well below $100 \mathrm{MeV}$, in sharp contrast with values around $0.5 \mathrm{GeV}$ extracted from lattice calculations. Despite the FCM is in good agreement with full QCD lattice data and is a well defined theoretical approach where confinement is, ab initio, the crucial dynamical aspect, some refinements seem to be needed once the astrophysical data are considered.

\section{References}

[1] M. Buballa, Phys. Rep. 407 (2005) 205.

[2] C. Maieron, M. Baldo, G. F. Burgio, and H.-J. Schulze, Phys. Rev. D 70, (2004) 043010.

[3] A. Di Giacomo, H.G. Dosch, V.I.Shevchenko and Y.A. Simonov, Phys. Rep 372, (2002) 319.

[4] M. Baldo, G.F. Burgio, P. Castorina, S. Plumari, and D. Zappalà, Phys. Rev. C75, (2007) 035804.

[5] M. Baldo, G.F. Burgio, P. Castorina, S. Plumari, and D. Zappalà, Phys. Rev. D78, (2008) 063009.

[6] M. Baldo, Nucl. Phys. A782c, (2007) 410, and references therein.

[7] J. Carlson, V. R. Pandharipande, and R. B. Wiringa, Nucl. Phys. A401, 59 (1983).

[8] M. Baldo, I. Bombaci, and G. F. Burgio, Astron. Astrophys. 328, (1997) 274.

[9] Yu.A. Simonov, Phys. Lett. B619, (2005) 293.

[10] Yu.A. Simonov, and M.A. Trusov, JETP Lett. 85 (2007) 598.

[11] Yu.A. Simonov, and M.A. Trusov, Phys. Lett. B650 (2007) 36.

[12] M.A. Shifman, A.I. Vainshtein, V.I. Zakharov, Nucl. Phys. B147 (1979) 448.

[13] E.V.Komarov, Yu.A.Simonov, Annals Phys. 323 (2008) 783.

[14] E.V. Komarov, and Yu.A. Simonov, Theory of Quark-Gluon Plasma and Phase Transition, arXiv:0801.2251.

[15] S.L. Shapiro and S.A. Teukolsky, Black Holes, White Dwarfs and Neutron Stars (John Wiley and Sons, New York, 1983).

[16] R. A. Hulse and J. H. Taylor, Astrophys. J. 195, (1975) L51.

[17] H. Quaintrell et al., Astron. Astrophys. 401, (2003) 313. 